\title{
Radiobiology of brain metastasis: applications in stereotactic radiosurgery
}

\author{
Jay Jagannathan, M.D., 'Jonathan H. Sherman, M.D., ${ }^{1}$ Gautam U. Mehta, M.S., 2 \\ and LaWrence S. Chin, M.D. ${ }^{3}$
}
${ }^{1}$ Department of Neurological Surgery, University of Virginia Health System, Charlottesville, Virginia; ${ }^{2}$ Georgetown University School of Medicine, Washington, DC; and ${ }^{3}$ Department of Neurological Surgery, Boston University Medical Center, Boston, Massachusetts

\begin{abstract}
$\checkmark$ Stereotactic radiosurgery is a neurosurgical modality in which a target lesion can be irradiated while sparing normal brain tissue. In some respects, brain metastasis is well suited for radiosurgery, as metastatic lesions tend to be small and well circumscribed and displace (but do not infiltrate) normal brain tissue, facilitating the delivery of radiation. Advances in stereotactic radiosurgical planning, such as blocking patterns and beam shaping, have allowed further targeting of discrete lesions while minimizing the effect of radiation toxicity on the central nervous system. In this paper the authors review the radiobiology of brain metastases and stereotactic radiosurgical approaches that can be used to treat these tumors safely.
\end{abstract}

KEY WORDS • brain metastases • radiobiology • radiosurgery • stereotaxy

F IRST INTRODUCED by Lars Leksell in 1951, SRS is a neurosurgical modality that combines stereotactic technique with highly focused high-energy radiation treatments, making it possible to deliver large doses of radiation to an extremely small target. ${ }^{22,34}$ By keeping individual doses of radiation small, normal brain parenchyma is protected while allowing a large dose of radiation to be delivered to the desired target.

In many respects, areas of brain metastasis are ideally suited for radiosurgery. ${ }^{24}$ These neoplasms tend to be small, have clear margins with respect to normal brain tissue, and are spherical or spheroid in shape, permitting treatment through single or multiple fractions. ${ }^{7}$ Published papers on SRS for brain metastasis have shown a control rate ranging from 85 to $95 \% .^{1,16-19}$ Good local tumor control and median survival rates comparable to that of resection followed by fractionated radiotherapy have been reported following SRS. ${ }^{10,18-20,31,40}$ Study results have also shown that patients with one or two brain metastases treated by radiosurgery have prolonged survival periods similar to those achieved using resection..$^{9,36}$

In recent years, the development of hyperfractionated radiosurgery, which is thought to reduce risks of patient morbidity, as well as frameless image-guided radiosur-

Abbreviations used in this paper: $\mathrm{CNS}=$ central nervous system; $\mathrm{MTD}=$ maximum tolerated dose; SRS = stereotactic radiosurgery. gery, which allows treatment of extracranial lesions, has further increased the scope of radiosurgery in the treatment of brain and spinal metastases. As the role of radiosurgery evolves in the treatment of brain metastasis, neurosurgeons will likely continue to be gatekeepers in deciding which patients will benefit from radiosurgery, conventional radiation treatment, open surgery, or other combinations of these modalities. To understand the rationale for these decisions fully, it is important to understand the biology of brain metastasis and the factors that make a lesion amenable to radiation treatment. In this paper we review the basic concepts of radiobiology and their use in the treatment of brain metastasis.

\section{TyPes OF IONIZING RADIATION}

The biological effectiveness of $\mathrm{x}$-rays, gamma rays, and protons is roughly equivalent, and each is considered to be a form of low linear energy transfer radiation. The use of protons in radiosurgery is based on the physical properties of these particles and the characteristics of dose deposition in irradiated tissues. ${ }^{32,33}$ Protons have a slightly higher radiobiological effectiveness than cobalt-60, which is a common source of gamma radiation. In practice, an adjustment is made for this small difference by calculating the dose for protons in cobalt Gray equivalents both in single and multiple fractions. 
Dose deposition is characterized by the Bragg peak, which is used in deciding treatment regimens that involve use of the proton beam. Qualitatively, the entrance dose for particle beams is relatively low compared with that for photons. An unaltered beam deposits more than $50 \%$ of its energy over a 2- to 3-cm narrow path at a depth in water that depends on the beam energy. The beam may be altered to spread the Bragg peak to conform to the thickness and depth of the volume to be treated. However, the entrance dose is significantly increased in this case. ${ }^{3}$

Gamma rays and $\mathrm{x}$-rays are forms of electromagnetic radiation whose energy ranges from 10.0 to $10.2 \times 10^{10}$ electron volts. The $x$-rays are produced when electrons in the outer shell of heavy atoms fall from a high to a low energy level and are thus produced extranuclearly. These $\mathrm{x}$-rays may be the product of radioactivity or created by human intervention using x-ray tubes or a linear accelerator, which accelerates electrons on a heavy metal target, producing a continuous spectrum of photon energies.

Gamma rays are photons emitted by radioactive nuclei and have a narrow range of energies $(10 \mathrm{keV}-10 \mathrm{MeV})$. Gamma rays and $\mathrm{x}$-rays are otherwise identical. The most common source of gamma rays used in radiotherapy is cobalt-60, which is commercially produced from cobalt59 and undergoes beta decay with a half-life of 5.27 years. Two gamma energies are emitted-1.17 and 1.33 $\mathrm{MeV}^{46}$ - with an effective average energy of $1.21 \mathrm{MeV}$. It is the subsequent gamma emission that makes it applicable to SRS. ${ }^{32,33}$

When cells are irradiated using low linear energy transfer radiation, most photons interact with water molecules by stripping an electron from a hydrogen atom, resulting in a fast electron and an ionized water molecule through scattering. The resulting fast electrons further interact with water molecules through further ionizing events, resulting in a positively charged water molecule that exhibits a short half-life before dissociating into an $\mathrm{H}^{+}$ion and an $\mathrm{OH}^{-}$free hydroxyl radical. The hydroxyl radical is reactive and has sufficient energy to break chemical bonds in nearby molecules. This indirect effect of radiation through a free radical intermediary is responsible for the majority $(>70 \%)$ of radiation-induced damage. The remaining damage is attributable to the direct effect of fast electrons interacting with biologically important molecules (that is, DNA). ${ }^{12}$

\section{Principles of RADiobiology}

Malignant tumors usually contain a proportion of hypoxic cells, which are often resistant to damage by $\mathrm{x}-$ rays or gamma rays. ${ }^{25}$ Cell survival after a single dose of ionizing radiation is a probability function measured using the Gray unit (absorption of $1 \mathbf{J}$ of radiation energy by 1 $\mathrm{kg}$ of tissue). Studies on tumor cell lines have shown that the apparent radiosensitivity of cultured tumor cell lines depends heavily on the culture conditions and the assays used to determine cell survival. ${ }^{26,27}$ In assessing cell survival, there appears to be a point at which the accumulation of sublethal insults at low doses has a cumulative effect. This may be a result of the fact that DNA is the target for cellular damage by ionizing radiation, and a doublestranded break may result in cell death. Double-stranded breaks may be a result of a single particle or the interac-

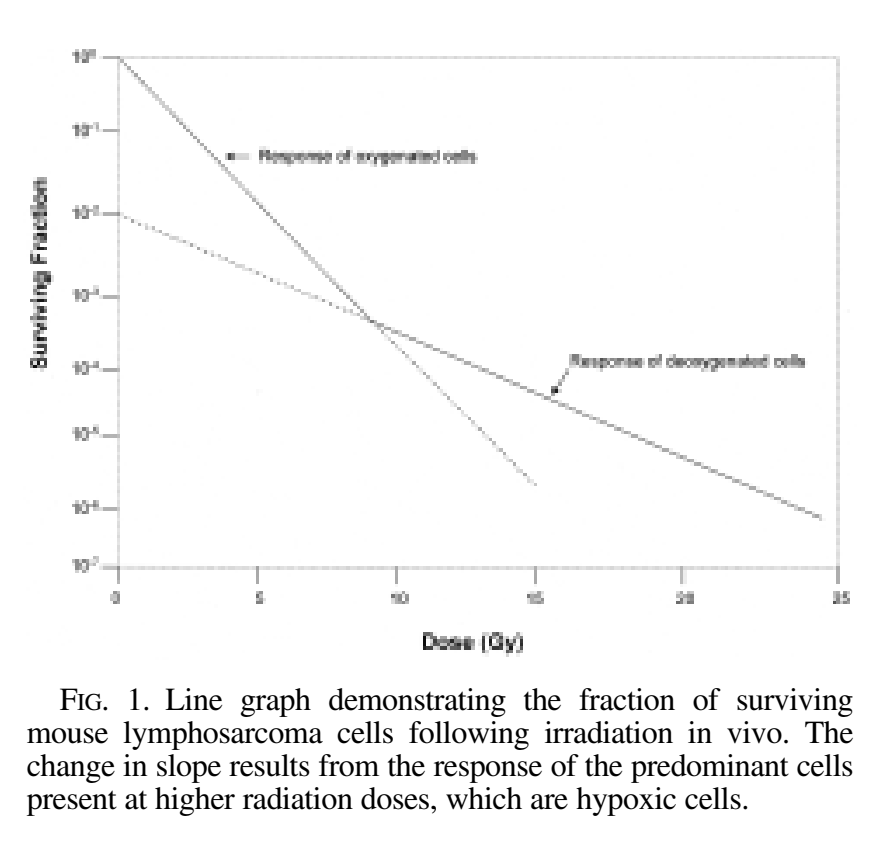

tion of two single-stranded breaks caused by separate particles occurring at close temporal and spatial distances. ${ }^{5}$

Typically, mammalian tumor cell survival curves obtained after a single dose of radiation have a characteristic shape, which is depicted in Fig. 1. There is generally a change in cell viability (often by a factor of 2.5-3) following radiation treatment, in which aerated cells become nonviable and the site of irradiation is dominated by hypoxic cells. From this observation, it is thus important to note that treatment of a tumor with a single large dose (tens of Gray) of radiation is often futile, as hypoxic cells are not adequately depopulated.

In spite of this, some groups have demonstrated a phenomenon known as reoxygenation, whereby tumors may reestablish their original pattern and proportion of oxygenated and hypoxic cells between sessions in which they receive fractionated doses of x-rays or gamma rays. In a fractionation regimen, therefore, each dose of radiation kills a proportion of cells and the interval between treatments allows hypoxic cells to reestablish their oxygenated state..$^{38}$

Another noteworthy radiobiological principle arises from experimental evidence indicating that there is a difference in shape between the dose-response relationship curve characteristic of early-responding tissues (including tumors) and that characteristic of late-responding tissues. ${ }^{42}$ The inference from experiments in animals, which has been confirmed in clinical practice, is that the doseresponse curve for late-responding tissue diminishes more rapidly than that for early-responding tissue. In mathematical terms, if the dose-response relationship is expressed in terms of a linear quadratic equation (see Wilder and colleagues ${ }^{42}$ ) in which cell survival ( $S$ ) is $S=\exp -$ $\alpha \mathrm{D}-\beta \mathrm{D}^{2}$ ("exp" represents exponential, " $\alpha$ " and " $\beta$ " are constants, and " $D$ " represents dose), the $\alpha / \beta$ ratio in the linear quadratic survival model shows that the dose needed to kill cells is small ( $<3 \mathrm{~Gy}$ ) for late-responding tissues and larger $(>8 \mathrm{~Gy})$ for early-responding tissues. ${ }^{4,37}$

The practical outcome of this difference in the shape of the dose-response curve is a marked difference in the res- 
ponse to fractionation in these two types of tissues. Lateresponding tissues are more sensitive to fractionation than early-responding tissues. Nevertheless, late-responding normal tissues are more likely to be spared when a fractionated treatment regimen is used than when a single acute dose is delivered for a given level of tumor damage. ${ }^{43}$

Providing sufficient time between fractions allows maximal recovery from sublethal damage, which is critical because sparing normal tissue is crucial in radiosurgery. The best information on the kinetics of repair of sublethal radiation damage in the CNS is found in the work of Ang and colleagues, ${ }^{2}$ who found half-times of 0.7 and 3.8 hours, respectively, for the fast and slow components of repair.

\section{Radiation In THE Treatment of Brain Metastasis}

Malignant tumors generally fall into the category of early-responding tissue containing hypoxic cells, whereas normal brain tissue consists primarily of late-responding tissues containing well-aerated cells. The efficacy of radiation treatment for brain metastasis can be greatly improved by fractionation, when compared with a single large radiation dose. Fractionation increases the cellular depopulation of a tumor for a given total radiation dose because of the phenomenon of reoxygenation. At the same time, fractionation reduces the damage to critical late-responding normal tissues, as shown by differences in the shape of the survival curve for late-responding tissues and by greater sensitivity to changes in fractionation. ${ }^{15,28}$ This phenomenon is in contrast to the treatment of many benign tumors and arteriovenous malformations, in which both targeted abnormal tissue and normal brain tissue consist of late-responding tissue of similar radiological types. There is little to be gained by fractionation in this situation. ${ }^{35}$

A large region of metastasis can also benefit from fractionation. As larger lesions have been associated with reduced MTDs, treatment often results in less effective local control. ${ }^{6,30}$ Fractionated therapy has been shown to permit more aggressive irradiation without an unacceptable increase in toxicity. ${ }^{23}$

The number of metastatic lesions has also been thought to influence the dose of radiation that can be tolerated, as cumulative targeting of multiple targets may lead to dangerous levels of radiation. However, authors of several studies have calculated this cumulative radiation to be within an acceptable range. ${ }^{44,45}$

\section{Radiosurgical Treatment Schemes for Brain Metastasis}

In the treatment of brain metastasis, various radiosurgical modalities-Gamma Knife, linear accelerator, and proton beam-achieve their effects by treating a discrete tumor with a high volume of radiation. ${ }^{8}$ Because earlyresponding tissue (the tumor) has a relatively high $\alpha / \beta$ ratio, it is relatively insensitive to fractionation and a single treatment is possible. Furthermore, unlike gliomas, the vast majority of brain metastases displace normal brain tissue, rather than infiltrate it, and provide a distinct bor- der that is useful for radiosurgical treatment.

The reason why the rules of fractionation can be violated in radiosurgery is because radiosurgery allows the surgeon to isolate the tumor from normal brain tissue spatially by prescribing a rapid dose falloff. In select cases, however, fractionated radiosurgery can be performed through external stereotactic treatment or by using an interstitial implant. Interstitial implant radiosurgery is performed by placing temporary or permanent low-dose-rate, high-activity ${ }^{125} \mathrm{I}$ implants around the area containing the surgically accessible lesion. ${ }^{11}$ Although effective in some cases, the major disadvantage of this technique is the possibility of rapid tumor shrinkage during the irradiation period, which could result in significant damage to normal brain tissue. ${ }^{21}$

An alternative approach is to use fractionated externalbeam radiosurgery. The fractionated external-beam technique allows for corrections to be made in the treatment regimen as the tumor shrinks, and for most patients it is easily performed during a short hospital stay. Generally speaking, the number of fractions used for the radiosurgical treatment of brain metastasis is significantly less than the 30 or more fractions associated with conventional radiotherapy. Using a small number of fractions is important because many types of brain metastasis (such as melanoma metastasis) are radioresistant and analysis has shown that they exhibit a large shoulder on the dose-survival curve). The use of a small number of fractions supports one of the potential advantages of radiosurgery (that large fractions are more effective at killing radioresistant tumors) while adding to the advantages of fractionation described earlier in this paper.

\section{Effects of Radiation on Normal Brain Tissue}

Normal brain tissue can be viewed as late-responding tissue (low $\alpha / \beta$ ratio) and is thus sensitive to radiation effects in large doses; this raises the concern about radiation necrosis during radiosurgery treatment planning. ${ }^{8}$ Evidence in experimental animal models, including nonhuman primates, has shown that increasing the volume to be irradiated to encompass normal brain or spinal cord tissue lowers the threshold of neuronal injury in the region and increases the slope of the dose-response curve to radiation, effectively causing more tissue injury. ${ }^{13}$ The volume effects of radiation are more pronounced in areas receiving high doses of radiation than in those receiving lower does.

Although treatment strategies, such as blocking patterns (described later in this section) and fractionation to effectuate dose delivery while sparing normal tissue, theoretically lower the risk of radiation-induced damage following radiosurgery, recent work has shown that the volume of brain tissue irradiated is correlated to the future development of radiation-induced toxicity. ${ }^{29,30}$ The Radiation Therapy and Oncology Group examined the MTD of single-fraction radiosurgery as a function of the total irradiated volume and established single-dose (margin) MTDs for volumes less than $4.2 \mathrm{~cm}^{3}$, from 4.2 to $14 \mathrm{~cm}^{3}$, and from 14.1 to $31 \mathrm{~cm}^{3}$. These MTDs are 24, 18, and $15 \mathrm{~Gy}$, respectively. When delivered in 2-Gy fractions, the equivalent doses are 64, 90, and $156 \mathrm{~Gy}$, respectively. ${ }^{30}$ 
There is evidence that repair of radiation-induced damage in the CNS occurs, particularly with respect to damage involving the spinal cord or the optic apparatus..$^{14,39}$ This phenomenon is most likely to be at least partially attributable to a repopulation of normal cells from surviving stem-cell populations or migration of cells from normal, nonirradiated tissues. These issues are particularly relevant in decisions of whether and when to again irradiate a region in a patient with evidence of tumor recurrence. However, most of the evidence regarding repeated irradiation of a CNS metastasis comes from an extrapolation from preclinical and retrospective clinical data and should be weighed carefully with alternatives to radiation and the risk of permanent sequelae after repeated radiosurgery. ${ }^{29}$

\section{Approaches to Minimize Toxicity in the CNS}

The best strategy to minimize radiation-induced CNS toxicity is always to minimize the dose administered to and the volume of the irradiated tissue. Careful treatment planning based on findings on modern modalities of imaging, such as computed tomography and magnetic resonance imaging, effective patient immobilization and fixation, and accurate treatment delivery all contribute to minimizing the size of the treated volumes and minimizing the dose delivered to normal structures. Dose-volume histogram analysis is also an essential element in optimizing radiosurgical treatment planning. Emerging technologies such as inverse treatment planning and intensitymodulated radiotherapy have also contributed to a reduction in the volume of normal tissue that is irradiated as well as reductions in the dose per fraction and the total dose. ${ }^{8}$

Development of blocking patterns is an established method of refining radiosurgery treatment, offering improved conformity and allowing the surgeon to adjust the peripheral isodose to the tumor margins. Standardized blocking patterns can significantly reduce the exposure to radiation, while increasing the conformity of the treatment plan, even for tumors with irregular margins. ${ }^{41}$

Single-dose radiosurgery may be preferable in cases in which the tumor volume is small and normal tissue tolerance is well respected. In other situations, however, in which normal structures (that is, the optic chiasm, brainstem, and spinal cord) lie adjacent to the tumor or in cases in which the tumor is large, dose fractionation may be the only method to provide safe and efficacious treatment. A specific study of cellular mechanisms of cell damage and repair following CNS irradiation is needed to improve our understanding of other ways in which we may protect the CNS from radiation-induced injury.

\section{Conclusions}

Brain metastases are well suited for radiosurgery because of their relatively small size and shape and location at the gray-white matter junction. The growth characteristics, oxygenation, and mitotic activity of metastasis in the brain are all major factors in determining the biological effectiveness of radiosurgery in treating metastatic disease. Although the incidence of radiation-induced damage following radiosurgery is low, experimental studies have shown a relationship between both the radiosurgical dose and volume treated and the extent of damage to normal tissue. Some of these problems can be reduced by the development of blocking patterns and by dose fractionation.

\section{References}

1. Alexander E III, Moriarty TM, Davis RB, Wen PY, Fine HA, Black PM, et al: Stereotactic radiosurgery for the definitive, noninvasive treatment of brain metastases. J Natl Cancer Inst 87:34-40, 1995

2. Ang KK, Price RE, Stephens LC, Jiang GL, Feng Y, Schultheiss TE, et al: The tolerance of primate spinal cord to re-irradiation. Int J Radiat Oncol Biol Phys 25:459-464, 1993

3. Austin-Seymour M, Munzenrider J, Linggood R, Goitein M, Verhey L, Urie M, et al: Fractionated proton radiation therapy of cranial and intracranial tumors. Am J Clin Oncol 13: 327-330, 1990

4. Bentzen SM, Turesson I, Thames HD: Fractionation sensitivity and latency of telangiectasia after postmastectomy radiotherapy: a graded-response analysis. Radiother Oncol 18:95-106, 1990

5. Brenner DJ, Hall EJ: Microbeams: a potent mix of physics and biology. Summary of the 5th International Workshop on Microbeam Probes of Cellular Radiation Response. Radiat Prot Dosimetry 99:283-286, 2002

6. Chang EL, Hassenbusch S Jr, Shiu AS, Lang FF, Allen PK, Sawaya $\mathrm{R}$, et al: The role of tumor size in the radiosurgical management of patients with ambiguous brain metastases. Neurosurgery 53:272-280, 2003

7. Chang SD, Adler JR Jr, Hancock SL: Clinical uses of radiosurgery. Oncology (Williston Park) 12:1181-1188, 1191-1192, 1998

8. Chin LS, Ma L, DiBiase S: Radiation necrosis following gamma knife surgery: a case-controlled comparison of treatment parameters and long-term clinical follow-up. J Neurosurg 94:899-904, 2001

9. Ferrara M, Bizzozzero L, Talamonti G, D’Angelo VA: Surgical treatment of 100 single brain metastases. Analysis of the results. J Neurosurg Sci 34:303-308, 1990

10. Flickinger JC, Lunsford LD, Somaza S, Kondziolka D: Radiosurgery: its role in brain metastasis management. Neurosurg Clin N Am 7:497-504, 1996

11. Gutin PH, Prados MD, Phillips TL, Wara WM, Larson DA, Leibel SA, et al: External irradiation followed by an interstitial high activity iodine-125 implant "boost" in the initial treatment of malignant gliomas: NCOG study 6G-82-2. Int J Radiat Oncol Biol Phys 21:601-606, 1991

12. Hall EJ, Brenner DJ: The radiobiology of radiosurgery: rationale for different treatment regimes for AVMs and malignancies. Int J Radiat Oncol Biol Phys 25:381-385, 1993

13. Hopewell JW, Morris AD, Dixon-Brown A: The influence of field size on the late tolerance of the rat spinal cord to single doses of X rays. Br J Radiol 60:1099-1108, 1987

14. Hornsey S, Myers R, Warren P: Residual injury in the spinal cord after treatment with $X$ rays or neutrons. Br J Radiol 55: 516-519, 1982

15. Houdek PV, Landy HJ, Quencer RM, Sattin W, Poole CA, Green BA, et al: MR characterization of brain and brain tumor response to radiotherapy. Int J Radiat Oncol Biol Phys 15:213-218, 1988

16. Hover KH, Hesse BM, Oetzel D, Rhein B, Lorenz WJ, Engelhart R, et al: [Comparison of dosimetric properties of 15$\mathrm{mV}$-photons and 14-meV-neutrons in external stereotaxic convergence therapy.] Strahlenther Onkol 167:651-657, 1991

17. Joseph J, Adler JR, Cox RS, Hancock SL: Linear accelerator- 
based stereotaxic radiosurgery for brain metastases: the influence of number of lesions on survival. J Clin Oncol 14: 1085-1092, 1996

18. Kihlstrom L, Karlsson B, Lindquist C: Gamma Knife surgery for cerebral metastases. Implications for survival based on 16 years experience. Stereotact Funct Neurosurg 61 (1 Supp):45-50, 1993

19. Kihlstrom L, Karlsson B, Lindquist C, Noren G, Rahn T: Gamma knife surgery for cerebral metastasis. Acta Neurochir Suppl 52 (Wien):87-89, 1991

20. Kondziolka D, Martin JJ, Flickinger JC, Friedland DM, Brufsky AM, Baar J, et al: Long-term survivors after gamma knife radiosurgery for brain metastases. Cancer 104:2784-2791, 2005

21. Leibel SA, Gutin PH, Wara WM, Silver PS, Larson DA, Edwards MS, et al: Survival and quality of life after interstitial implantation of removable high-activity iodine-125 sources for the treatment of patients with recurrent malignant gliomas. Int J Radiat Oncol Biol Phys 17:1129-1139, 1989

22. Leksell L: Stereotactic radiosurgery. J Neurol Neurosurg Psychiatry 46:797-803, 1983

23. Nishizaki T, Saito K, Jimi Y, Harada N, Kajiwara K, Nomura $\mathrm{S}$, et al: The role of cyberknife radiosurgery/radiotherapy for brain metastases of multiple or large-size tumors. Minim Invasive Neurosurg 49:203-209, 2006

24. Noel G, Boisserie G, Feuvret L, Mazeron JJ: [Radiosurgery of brain metastasis: reflexions, controversies and unanswered questions in 2004.] Bull Cancer 91:81-93, 2004 (Fr)

25. Powers WE, Tolmach LJ: Demonstration of an anoxic component in a mouse tumor-cell population by in vivo assay of survival following irradiation. Radiology 83:328-336, 1964

26. Powers WE, Tolmach LJ: A multicomponent x-ray survival curve for mouse lymphosarcoma cells irradiated in vivo. Nature 197:710-711, 1963

27. Powers WE, Tolmach LJ: Pre-operative radiation therapy: biological basis and experimental investigation. Nature 201:272-273, 1964

28. Schwade JG, Houdek PV, Landy HJ, Bujnoski JL, Lewin AA, Abitol AA, et al: Small-field stereotactic external-beam radiation therapy of intracranial lesions: fractionated treatment with a fixed-halo immobilization device. Radiology 176:563-565, 1990

29. Shaw E, Scott C, Souhami L, Dinapoli R, Bahary JP, Kline R, et al: Radiosurgery for the treatment of previously irradiated recurrent primary brain tumors and brain metastases: initial report of radiation therapy oncology group protocol (90-05). Int J Radiat Oncol Biol Phys 34:647-654, 1996

30. Shaw E, Scott C, Souhami L, Dinapoli R, Kline R, Loeffler J, et al: Single dose radiosurgical treatment of recurrent previously irradiated primary brain tumors and brain metastases: final report of RTOG protocol 90-05. Int J Radiat Oncol Biol Phys 47:291-298, 2000

31. Sheehan J, Kondziolka D, Flickinger J, Lunsford LD: Radiosurgery for patients with recurrent small cell lung carcinoma metastatic to the brain: outcomes and prognostic factors. J Neurosurg 102 (Suppl):247-254, 2005

32. Shrieve DC: Basic principles of radiobiology applied to radio- therapy of benign intracranial tumors. Neurosurg Clin N Am 17:67-78, 2006

33. Shrieve DC, Klish M, Wendland MM, Watson GA: Basic principles of radiobiology, radiotherapy, and radiosurgery. Neurosurg Clin N Am 15:467-479, 2004

34. Steiner L, Leksell L, Forster DM, Greitz T, Backlund EO: Stereotactic radiosurgery in intracranial arterio-venous malformations. Acta Neurochir Suppl (Wien) 21:195-209, 1974

35. Steiner L, Lindquist C, Adler JR, Torner JC, Alves W, Steiner M: Outcome of radiosurgery for cerebral AVM. J Neurosurg 77:823, 1992

36. Sundaresan N, Galicich JH: Surgical treatment of brain metastases. Clinical and computerized tomography evaluation of the results of treatment. Cancer 55:1382-1388, 1985

37. Thames HD, Bentzen SM, Turesson I, Overgaard M, van den Bogaert W: Fractionation parameters for human tissues and tumors. Int J Radiat Biol 56:701-710, 1989

38. Thames HD, Bentzen SM, Turesson I, Overgaard M, Van den Bogaert W: Time-dose factors in radiotherapy: a review of the human data. Radiother Oncol 19:219-235, 1990

39. Varlotto JM, Flickinger JC, Kondziolka D, Lunsford LD, Deutsch M: External beam irradiation of craniopharyngiomas: long-term analysis of tumor control and morbidity. Int J Radiat Oncol Biol Phys 54:492-499, 2002

40. Varlotto JM, Flickinger JC, Niranjan A, Bhatnagar A, Kondziolka D, Lunsford LD: The impact of whole-brain radiation therapy on the long-term control and morbidity of patients surviving more than one year after gamma knife radiosurgery for brain metastases. Int J Radiat Oncol Biol Phys 62: 1125-1132, 2005

41. Vaughan P, Hampshire A, Soanes T, Kemeny A, Radatz M, Rowe J, et al: The clinical application of plugging patterns for the Leksell gamma knife. J Neurosurg 97:579-581, 2002

42. Wilder RB, DeNardo GL, Sheri S, Fowler JF, Wessels BW, DeNardo SJ: Application of the linear-quadratic model to myelotoxicity associated with radioimmunotherapy. Eur J Nucl Med 23:953-957, 1996

43. Withers HR, Thames HD Jr, Flow BL, Mason KA, Hussey DH: The relationship of acute to late skin injury in 2 and 5 fraction/week gamma-ray therapy. Int J Radiat Oncol Biol Phys 4:595-601, 1978

44. Yamamoto M, Ide M, Nishio S, Urakawa Y: Gamma Knife radiosurgery for numerous brain metastases: is this a safe treatment? Int J Radiat Oncol Biol Phys 53:1279-1283, 2002

45. Yang CC, Ting J, Wu X, Markoe A: Dose volume histogram analysis of the gamma knife radiosurgery treating twenty-five metastatic intracranial tumors. Stereotact Funct Neurosurg 70:41-49, 1998

46. Zaider M, Brenner DJ, Hall EJ, Kliauga PJ: The link between physics and biology. Am J Clin Oncol 11:212-219, 1988

Manuscript submitted December 18, 2006

Accepted February 6, 2007.

Address reprint requests to: Jay Jagannathan, M.D., Department of Neurological Surgery, Box 800212, University of Virginia Health System, Charlottesville, Virginia 22908. email: jj5a@ virginia.edu. 